Communications in Physics, Vol. 28, No. 2 (2018), pp. 127-138

DOI:10.15625/0868-3166/28/2/12541

\title{
A COMPARATIVE STUDY OF OPTICAL BISTABILITY IN THREE-LEVEL EIT CONFIGURATIONS
}

\author{
LE VAN DOAI $^{a, \dagger}, \mathrm{LE} \mathrm{THI} \mathrm{MINH} \mathrm{PHUONG}^{b}, \mathrm{NGUYEN} \mathrm{TUAN} \mathrm{ANH}^{a, c}$, DOAN HOAI SON $^{d}$, \\ DINH XUAN KHOA ${ }^{a}$, VU NGOC SAU ${ }^{a}$ AND NGUYEN HUY BANG ${ }^{a}$ \\ ${ }^{a}$ Vinh University, 182 Le Duan Street, Vinh City, Vietnam \\ ${ }^{b}$ Sai Gon University, 273 An Duong Vuong street, Ho Chi Minh City, Vietnam \\ ${ }^{c}$ Ho Chi Minh City University of food industry, Ho Chi Minh City, Vietnam \\ ${ }^{d}$ Ha Tinh University, Ha Tinh Province, Vietnam \\ ${ }^{\dagger} E$-mail: doailv@vinhuni.edu.vn
}

Received 9 May 2018

Accepted for publication 29 May 2018

Published 16 June 2018

\begin{abstract}
We present a comparative study of optical bistability $(O B)$ in three-level atomic configurations, including $\Lambda$-, cascade-, and $V$-types under the conditions of electromagnetically induced transparency (EIT). In the steady regime, the input-output intensity relations for the $O B$ in each configuration have been derived in analytical form. The model allows one to construct a clear picture on how the threshold intensity, and other characteristics of the OB are continuously modified with respects to controllable parameters of the laser fields, cooperation parameter, and other physical parameters of atomic system. The results showed that the threshold intensity of $O B$ in $\Lambda$-type system is much less than the other ones and the threshold intensity of $O B$ in V-type system is the largest one. The analytical result is convenient to choose excitation configuration for experimental observations and related applications in photonic devices.
\end{abstract}

Keywords: optical bistability, effects of atomic coherence.

Classification numbers: 42.65.Pc; 42.50.Gy.

(C)2018 Vietnam Academy of Science and Technology 


\section{INTRODUCTION}

The OB is an interesting topic in nonlinear optics because of its potential applications in photonic devices, such as all-optical switches, memory elements, and all-optical logic gates and so on. In the early years of the OB research for atomic media, a great interest was focused on using two-level atomic systems [1-3]. The saturated absorption and intensity-dependent refractive index are attributed to two different mechanisms for absorptive $\mathrm{OB}$ and dispersive OB, respectively. It was shown that, the OB behavior occurs only in a certain domain of input intensity values; the threshold intensity as well as the width of OB is not controllable [2,3].

In recent years, the advent of the EIT [4] has provided a medium with zero absorption [4-7] and giant Kerr nonlinearity [8-10]. Indeed, thanks to such controllable optical properties of the EIT medium, both threshold intensity and width of OB can be simply controlled and reduced significantly. Basic configurations for the OB using the EIT medium are three-level atomic systems, including the $\Lambda$-, cascade- and V-type configurations. In each configuration, the EIT efficiency is different, in which the $\Lambda$ configuration is the best, whereas the V configuration is the worst. Therefore, the OB behaviors in each configuration are also different. Although properties of the three-level OB were studied numerically [11-20], numerical results do not show a continuous change in the input-output intensity versus laser parameters. Moreover, a comparison among the OB configurations is needed to choose an appropriate excitation configuration for experimental realizations.

In this work, an input-output intensity relation of OB is derived in analytical form for threelevel atomic media consisting of the $\Lambda$-, cascade- and V-type configurations under the steadyregime. Influences of intensity and frequency of the laser fields and cooperation parameter of

atomic medium on the $\mathrm{OB}$ are studied. Intensity thresholds of $\mathrm{OB}$ in three-level configurations are compared.

\section{MODEL OF ATOMIC OPTICAL BISTABILITY}

We consider a medium of length $L$ containing $N$ three-level atoms placed in a unidirectional ring cavity as shown in Fig. 1. For simplicity, we assume both mirrors M3 and M4 are perfect reflective whereas both mirrors M1 and M2 are the same, each has a reflectivity $R$ and transitivity $T$, with $R+T=1$. The three possible coupling configurations of three-level atomic system illustrated in Fig. 2, which includes $\Lambda$-, cascade- and V-type schemes. The strong coupling laser $\left(E_{c}\right)$ with frequency $\omega_{c}$ couples the transition $|2\rangle \leftrightarrow|3\rangle$ (for $\Lambda$ and cascade schemes) or $|1\rangle \leftrightarrow|3\rangle$ (for $\mathrm{V}$ scheme) and the week probe laser $\left(E_{p}\right)$ with frequency $\omega_{p}$ applies the transition $|1\rangle \leftrightarrow|2\rangle$. In the ring cavity configuration, only probe beam $E_{p}$ is circulated in the ring cavity but nor the coupling beam $E_{c}$

The total electromagnetic field can be written as

$$
E=E_{p} e^{-i \omega_{p} t}+E_{c} e^{-i \omega_{c} t}+c . c .,
$$

Propagation of probe laser field in the medium is governed by Maxwell's wave equation in the slowly varying envelop approximation as [2]:

$$
\frac{\partial E_{p}}{\partial t}+c \frac{\partial E_{p}}{\partial z}=i \frac{\omega_{p}}{2 \varepsilon_{0}} P\left(\omega_{p}\right),
$$




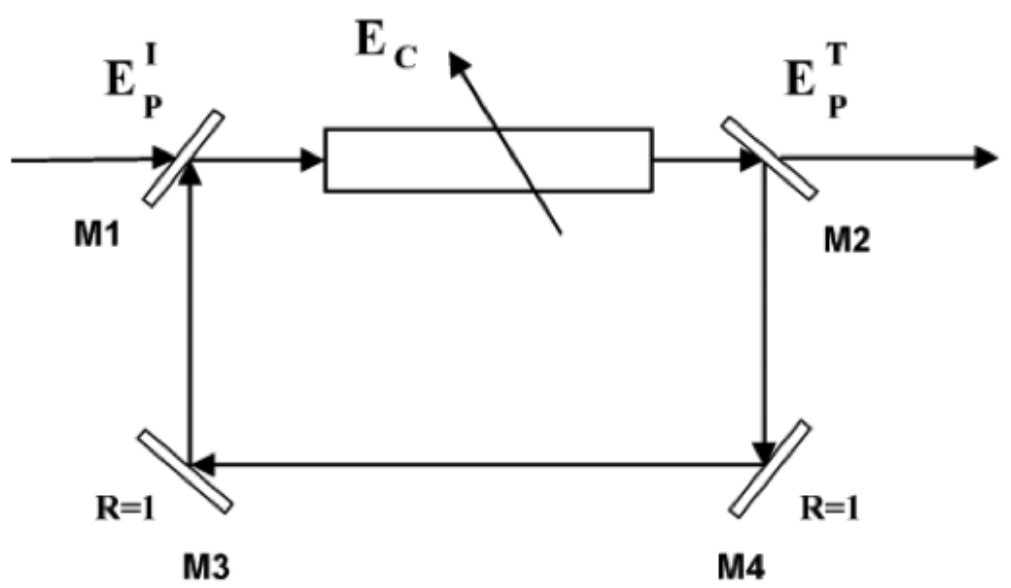

Fig. 1. Configuration of unidirectional ring cavity contains three-level atoms

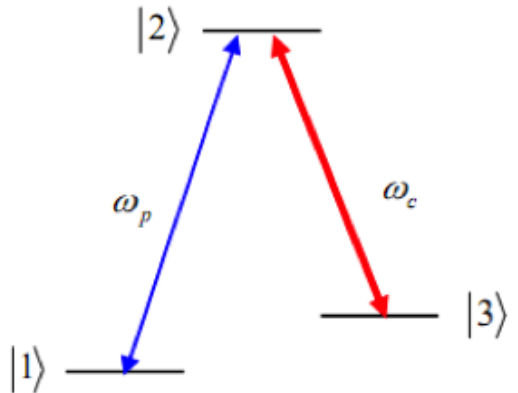

(a)

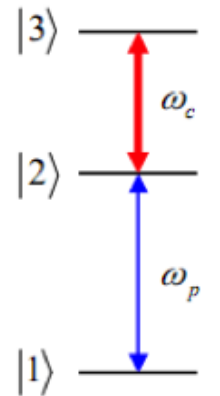

(b)

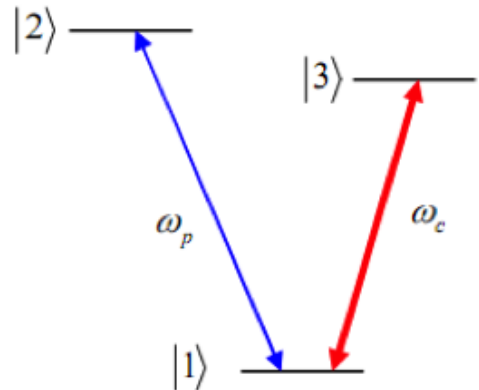

(c)

Fig. 2. Three-level excitation schemes: (a) $\Lambda$, (b) cascade, and (c) V.

where $c$ and $\varepsilon$ are the speed of light and permittivity of free space, respectively; $P\left(\omega_{p}\right)$ is the slowly oscillating term of the induced polarization in the transition $|1\rangle \leftrightarrow|2\rangle$ given by

$$
P\left(\omega_{p}\right)=N d_{21} \rho_{21}
$$

where $d_{21}$ denotes the electric dipole moment and $\rho_{21}$ is the corresponding density matrix element. Substituting Eq. (3) into Eq. (2), we have

$$
\frac{\partial E_{p}}{\partial z}=i \frac{N \omega_{p} d_{21}}{2 c \varepsilon_{0}} \rho_{21}
$$


For a perfectly tuned ring cavity, in the steady state limit, the boundary conditions imposed between the incident and transmitted probe fields are [2]:

$$
\begin{gathered}
E_{p}(L)=E_{p}^{T} / \sqrt{T}, \\
E_{p}(0)=\sqrt{T} E_{p}^{T}+R E_{p}(L) .
\end{gathered}
$$

The second term in the right-hand side of Eq. (6) denotes the feedback from the mirror, which is essential for the creation of OB. That is, if $R=0$ then no OB occurs

By normalizing the incident and transmitted probe field as

$$
Y=\frac{d_{21} E_{p}^{I}}{\hbar \sqrt{T}} \quad X=\frac{d_{21} E_{p}^{T}}{\hbar \sqrt{T}}
$$

the input-output intensity relation for the probe field becomes:

$$
Y=X-i C \rho_{21},
$$

where

$$
C=\frac{N \omega_{p} L d_{21}^{2}}{2 c \varepsilon_{0} \hbar T}
$$

is the cooperation parameter of the three-level atomic medium inside the ring cavity. From Eq. (8) we see that transmitted field depends on the incident probe field and the coherence term $\rho_{21}$ which are calculated from the Liouville equation as [20]:

$$
\dot{\rho}=-\frac{i}{\hbar}[H, \rho]+\Lambda \rho,
$$

where $H$ is the total Hamiltonian and $\Lambda \rho$ represents the decay part.

\section{ANALYSIS OF OPTICAL BISTABILITY BEHAVIOR}

\section{III.1. $\Lambda$-type system}

Firstly, we consider a $\Lambda$-type scheme as shown in Fig. 2a. The Rabi frequencies for the probe and coupling lasers are defined by

$$
\begin{aligned}
& \dot{\rho}_{11}=\Gamma_{31} \rho_{33}+\Gamma_{21} \rho_{22}+\frac{i}{2} \Omega_{p}\left(\rho_{21}-\rho_{12}\right) \\
& \dot{\rho}_{22}=-\left(\Gamma_{23}+\Gamma_{21}\right) \rho_{22}+\frac{i}{2} \Omega_{p}\left(\rho_{12}-\rho_{21}\right)+\frac{i}{2} \Omega_{c}\left(\rho_{32}-\rho_{23}\right) \\
& \dot{\rho}_{33}=-\Gamma_{31} \rho_{33}+\Gamma_{23} \rho_{22}-\frac{i}{2} \Omega_{c}\left(\rho_{32}^{-} \rho_{23}\right) \\
& \dot{\rho}_{21}=\bar{\gamma}_{21} \rho_{21}+\frac{i}{2} \Omega_{p}\left(\rho_{11}-\rho_{22}\right)+\frac{i}{2} \Omega_{c} \rho_{31} \\
& \dot{\rho}_{23}=\bar{\gamma}_{23} \rho_{23}+\frac{i}{2} \Omega_{c}\left(\rho_{33}-\rho_{22}\right)+\frac{i}{2} \Omega_{p} \rho_{13} \\
& \dot{\rho}_{31}=\bar{\gamma}_{31} \rho_{31}-\frac{i}{2} \Omega_{p} \rho_{32}+\frac{i}{2} \Omega_{c} \rho_{21} .
\end{aligned}
$$


The above equations are constrained by $\rho_{11}+\rho_{22}+\rho_{33}=1$ and $\rho_{k i}=\rho_{i k}^{*}$. Here, $\bar{\gamma}_{21}=$ $i \Delta_{p}-\gamma_{21}, \bar{\gamma}_{23}=i \Delta_{c}-\gamma_{23}$ and $\bar{\gamma}_{31}=i\left(\Delta_{p}-\Delta_{c}\right)-\gamma_{31} ; \gamma_{i k}$ is represented with the decay rates $\Gamma_{i k}$ from state $|i\rangle$ to $|j\rangle$ as $\gamma_{i k}=\frac{1}{2}\left(\sum_{E_{j}<E_{i}} \Gamma_{i j}+\sum_{E_{l}<E_{k}} \Gamma_{k l}\right)$

Now, we solve the density matrix equations from Eq. (11) to Eq. (16) in the steady regime to determine the coherence term $\rho_{21}$. From Eqs. (12), (13) and (16), we derive $\left(\rho_{33}-\rho_{22}\right)$ as following:

$$
\rho_{33}-\rho_{22}=\frac{i\left[\left(\Gamma_{23}-\Gamma_{31}\right) \Omega_{p}^{2}+\left(\Gamma_{21}+\Gamma_{31}\right) \Omega_{c}^{2}\right]}{2 \Gamma_{31}\left(\Gamma_{21}+\Gamma_{23}\right) \Omega_{p}}\left(\rho_{12}-\rho_{21}\right)-\frac{\left(\Gamma_{21}+\Gamma_{31}\right) \Omega_{c}}{\Gamma_{31}\left(\Gamma_{21}+\Gamma_{23}\right) \Omega_{p}}\left(\bar{\gamma}_{13} \rho_{13}+\bar{\gamma}_{31} \rho_{31}\right) .
$$

Substituting Eqs. (17) into Eq. (15) and after several calculations and rearrangements, we obtained:

$$
\begin{aligned}
& {\left[8 \Gamma_{31}\left(\Gamma_{21}+\Gamma_{23}\right) \bar{\gamma}_{23} \bar{\gamma}_{13}+2 \Gamma_{31}\left(\Gamma_{21}+\Gamma_{23}\right) \Omega_{p}^{2}-2\left(\Gamma_{21}+\Gamma_{31}\right) \bar{\gamma}_{13} \Omega_{c}^{2}\right] \rho_{13} } \\
= & 2\left(\Gamma_{21}+\Gamma_{31}\right) \bar{\gamma}_{31} \Omega_{c}^{2} \rho_{31}+4 i \Gamma_{31}\left(\Gamma_{21}+\Gamma_{23}\right) \bar{\gamma}_{23} \Omega_{c} \rho_{12} \\
& +i \Omega_{c}\left[\left(\Gamma_{23}-\Gamma_{31}\right) \Omega_{p}^{2}+\left(\Gamma_{21}+\Gamma_{31}\right) \Omega_{c}^{2}\right]\left(\rho_{21}-\rho_{12}\right) .
\end{aligned}
$$

We set

$$
\begin{aligned}
A & =\Omega_{c}\left[\left(\Gamma_{23}-\Gamma_{31}\right) \Omega_{p}^{2}+\left(\Gamma_{21}+\Gamma_{31}\right) \Omega_{c}^{2}\right], \\
A_{31} & =2\left(\Gamma_{21}+\Gamma_{31}\right) \bar{\gamma}_{31} \Omega_{c}^{2}, \\
A_{23} & =4 \Gamma_{31}\left(\Gamma_{21}+\Gamma_{23}\right) \bar{\gamma}_{23} \Omega_{c} \\
A_{123} & =8 \Gamma_{31}\left(\Gamma_{21}+\Gamma_{23}\right) \bar{\gamma}_{23} \bar{\gamma}_{13}+2 \Gamma_{31}\left(\Gamma_{21}+\Gamma_{23}\right) \Omega_{p}^{2}-2\left(\Gamma_{21}+\Gamma_{31}\right) \bar{\gamma}_{13} \Omega_{c}^{2} .
\end{aligned}
$$

Therefore, Eq. (18) becomes

$$
\rho_{31}=\frac{i\left(A A_{123}+A_{13} A-A_{32} A_{123}\right) \rho_{21}+i\left(A_{13} A_{23}-A A_{123}-A_{13} A\right) \rho_{12}}{A_{123} A_{321}-A_{13} A_{31}} .
$$

Substituting Eq. (19) into Eq. (15), we found the solution of the matrix element $\rho_{21}$ under the initial condition $\rho_{11}^{(0)} \approx 1, \rho_{22}^{(0)} \approx \rho_{33}^{(0)} \approx 0$ as follow:

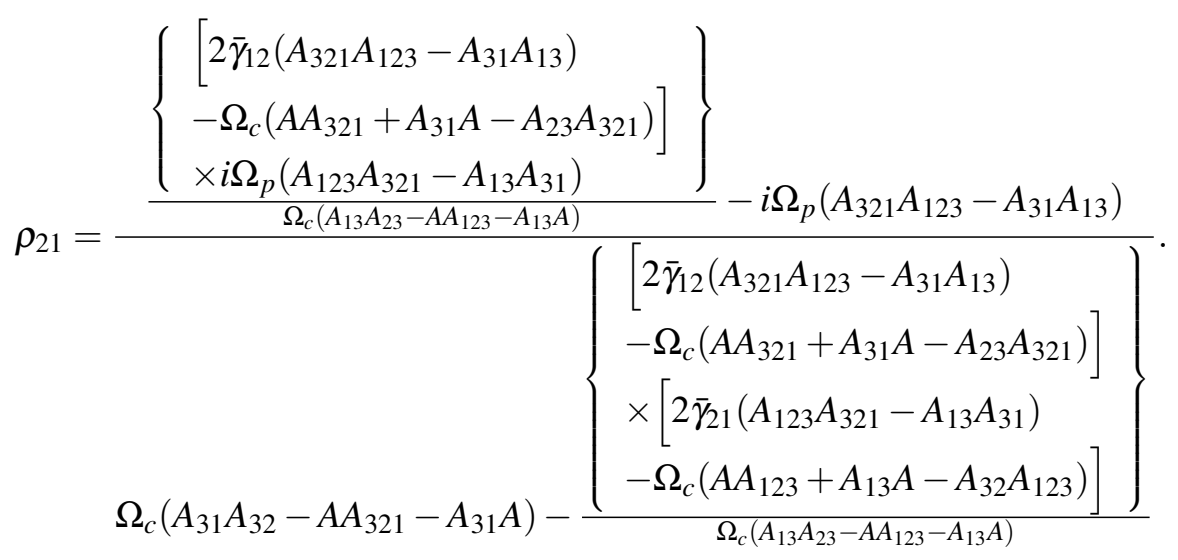


where $A_{k j i}$ and $\bar{\gamma}_{j i}$ are the complex conjugation of $A_{i j k}$ and $\bar{\gamma}_{i j}$, respectively. According to expressions (10) and (20), we can analyze the bistable behavior of medium which continuously modifies with respects to controllable parameters of the coupling laser, namely, frequency detuning and intensity. The result can generally be applied to atomic and molecular systems having spectroscopic structure similar to that presented in Fig.2a. Here, we applied to the case of ${ }^{85} \mathrm{Rb}$ atomic vapor with the states $|1\rangle,|2\rangle$ and $|3\rangle$ are chosen as $5 \mathrm{~S}_{1 / 2}(F=2), 5 \mathrm{~S}_{1 / 2}(F \prime=3)$ and $5 P_{3 / 2}\left(F^{\prime \prime}=3\right)$, respectively. The corresponding decay rates of ${ }^{85} \mathrm{Rb}$ atom are $\Gamma_{21}=\Gamma_{23}=6 \mathrm{MHz}=6 \gamma$ [21]. The advantage of $\Lambda$ configuration is the decoherence rate between states $|3\rangle$ and $|1\rangle$ which is very small $\gamma_{31}=0.03 \gamma$, therefore the EIT effect is the best [20].

We plot the OB versus the frequency probe detuning $\Delta_{p}$ when fixed the parameters of the coupling laser at [when the parameters of the coupling laser are fixed at] $\Delta_{c}=0$ and $\Omega_{c}=2 \gamma$. The surface plot of output-input fields versus $\Delta_{p}$ is represented in Fig. 3a. For a detail, we plot inputoutput intensity fields at several values of $\Delta_{p}$, as in Fig. 3(b). From Fig. 3, one can find [sees] that decreasing the frequency detuning leads to a decrease of the switching thresholds of OB because the Kerr nonlinearity of atomic medium is significantly enhanced in the resonant atomic vicinity under EIT conditions [7].
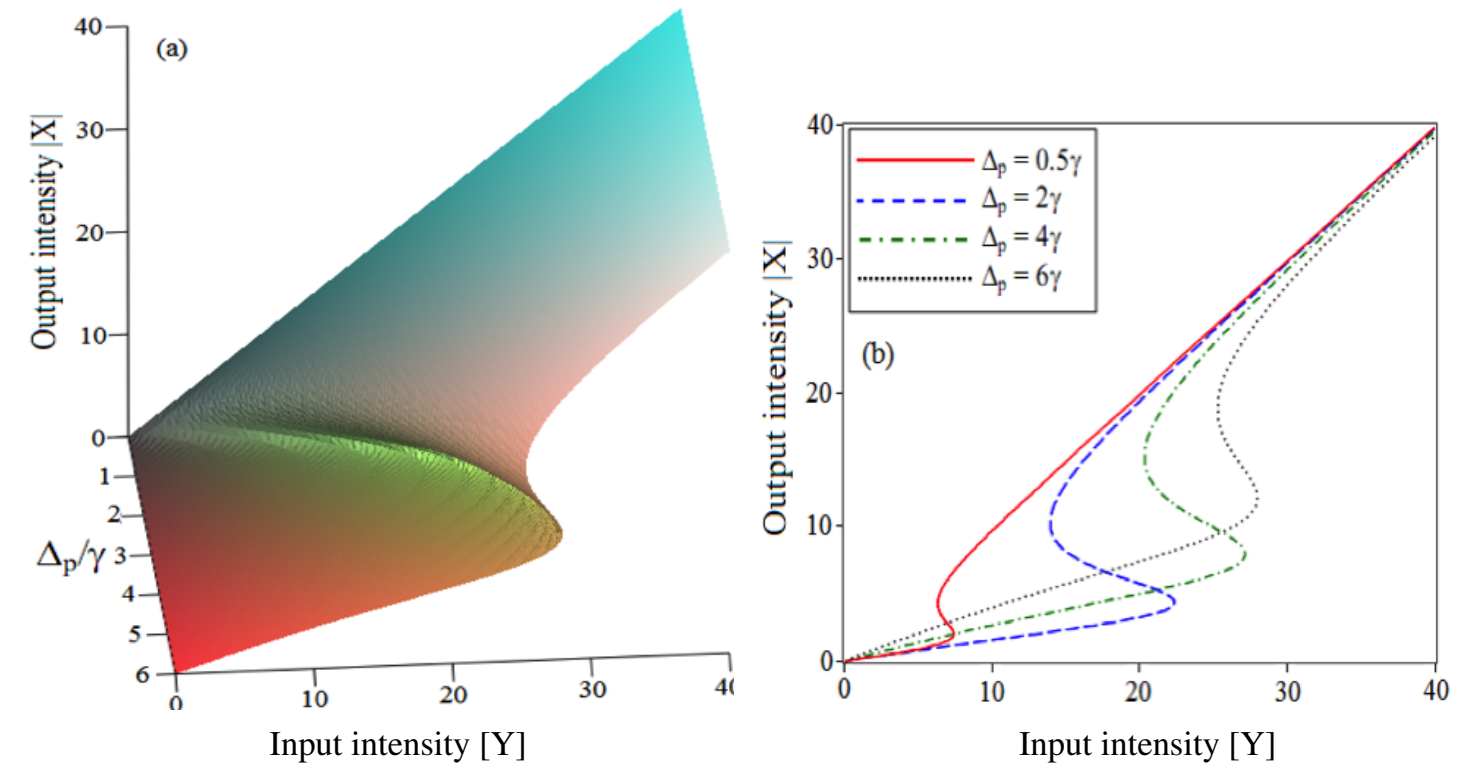

Fig. 3. (a) Surface plots of input-output fields versus frequency detuning $\Delta_{p}$ at fixed values $\Omega_{c}=2 \gamma, \Delta_{c}=0$, and $C=40 \gamma$. (b) represents particular case of Fig. 3(a) that illustrates dependence of switching thresholds on frequency detuning.

The influence of coupling laser intensity $\Omega_{c}$ on the OB behavior of system is displayed in Fig. 4. Here, values of frequency detuning of probe and coupling field are fixed at $\Delta_{p}=2 \gamma$ and $\Delta_{c}=0$, respectively. It is easily seen that both the switching thresholds and width of OB are decreased when increasing the Rabi frequency $\Omega_{c}$. A qualitative explanation for this phenomenon as [is as] follows: an increase in Rabi frequency reduces the absorption and simultaneously enhances Kerr nonlinearity which makes the cavity field easier to reach saturation. 

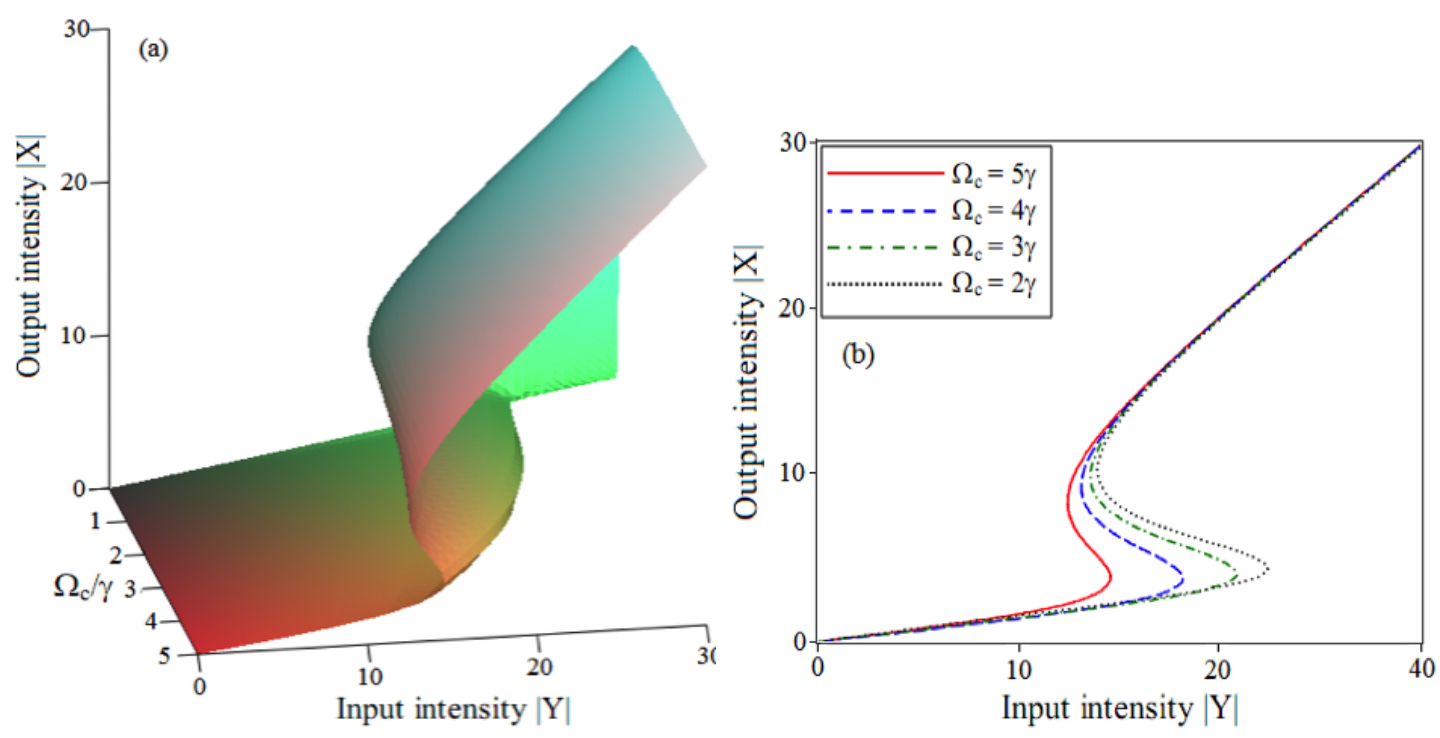

Fig. 4. (a) Surface plots of input-output fields versus the Rabi-frequency $\Omega_{c}$ at fixed values: $\Delta_{p}=2 \gamma, \Delta_{c}=0$, and $C=40 \gamma$. (b) represents particular case of Fig. 4(a) that illustrates dependence of switching thresholds on intensity of coupling field.
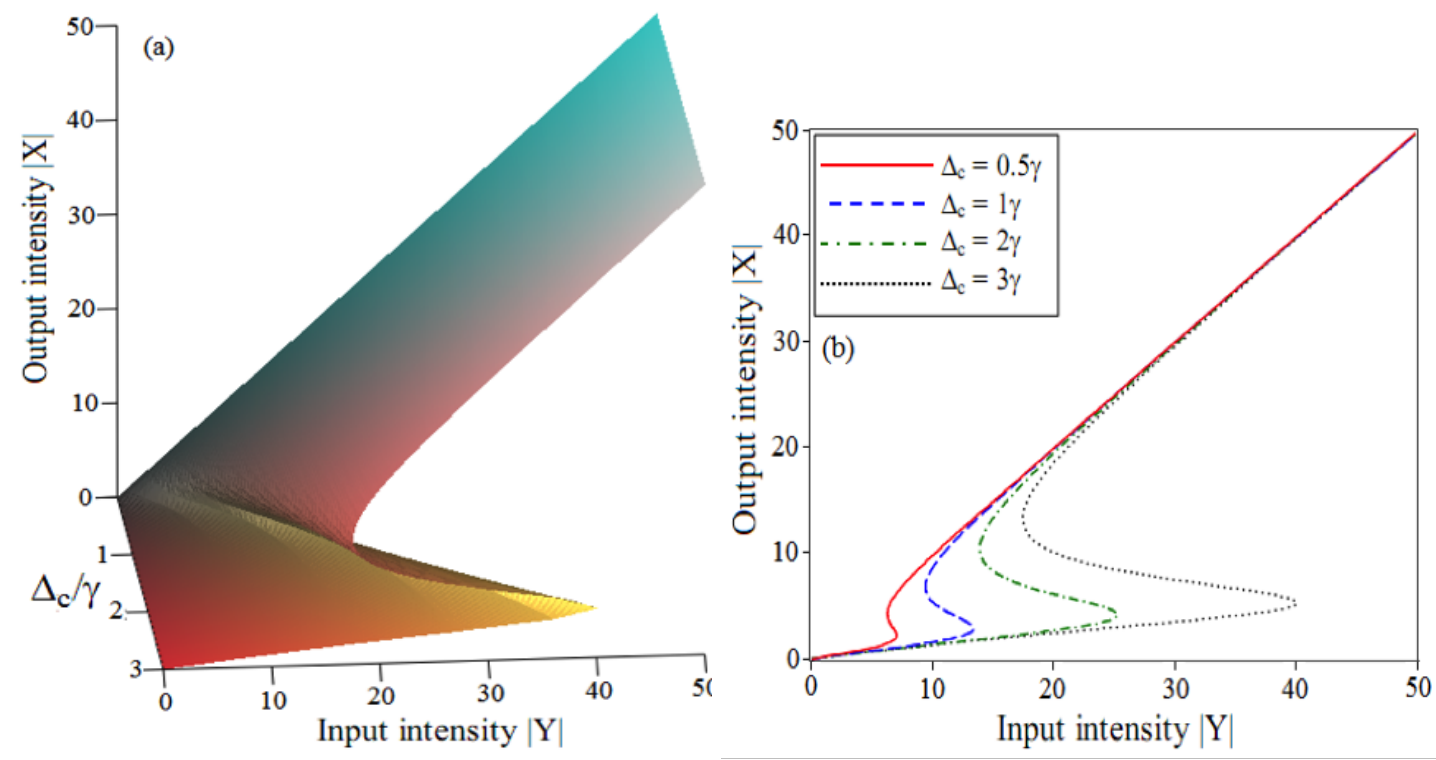

Fig. 5. (a) Surface plots of input-output intensity relation versus the frequency detuning $\Delta_{c}$ at fixed values: $\Delta_{p}=2 \gamma, \Omega_{c}=2 \gamma$, and $C=40 \gamma$. (b) represents particular case of Fig. 5(a) that illustrates dependence of switching thresholds on the frequency detuning of coupling field.

The influence of the coupling frequency detuning on OB behavior is plotted in Fig. 5. Here, other parameters are chosen as $\Delta_{p}=2 \gamma$ and $\Omega_{c}=2 \gamma$. The result in Fig. 5 shows that one may 
reduce both the threshold intensity and width of OB by tuning the coupling frequency detuning nearer resonance with the atomic frequency. The reason for this reduction is due to a sensitive dependence of Kerr nonlinearity on the coupling frequency detuning [7].

The influence of cooperation parameter $C$ on the OB is displayed in Fig. 6. Here, we fixed the parameters of probe and coupling laser at $\Delta_{p}=2 \gamma$ and $\Delta_{c}=0$, and $\Omega_{c}=2 \gamma$, respectively. It is clearly see [seen] that an increase in cooperative parameter leads to increasing the width and the threshold intensity of OB curve. Such behaviors are attributed to $C$ which is proportional to atomic density via $C=N \omega_{p} L d_{21}^{2} / 2 c \varepsilon_{0} \hbar T$. Physically, an increase in $C$ leads to an increase in absorption for the probe light, thus lowering the upper OB branch (output) and requiring higher switching threshold intensity.
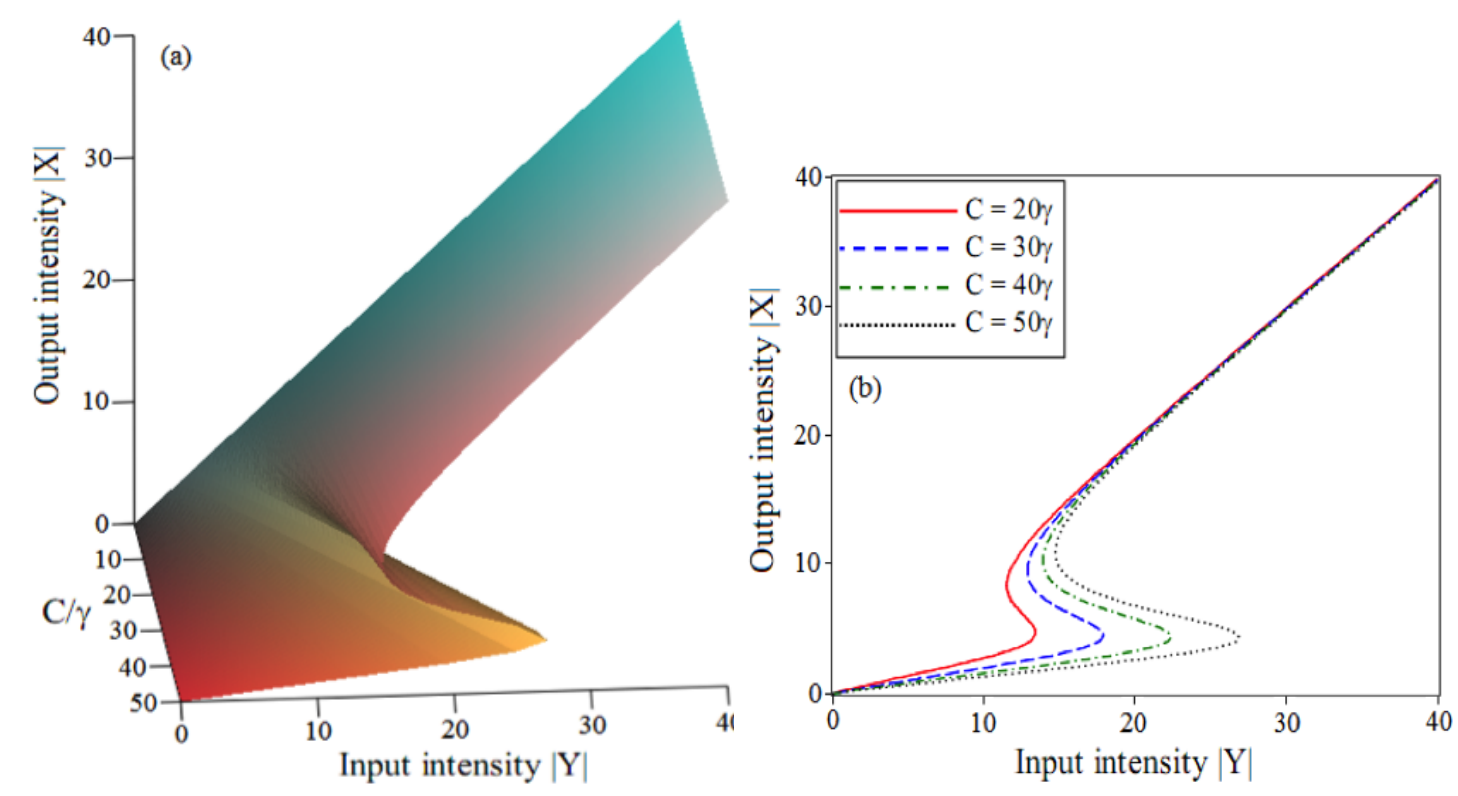

Fig. 6. (a) Surface plots of input-output intensity relation versus of cooperation parameter $C$ at fixed values: $\Delta_{p}=2 \gamma, \Delta_{c}=0$, and $\Omega_{c}=2 \gamma$. (b) represents particular case of Fig. 6(a) that illustrates dependence of switching thresholds on cooperation parameter $C$.

\section{III.2. Cascade-type system}

The cascade-type scheme considered here is shown in Fig. 2b. Accordingly, the systematic density matrix of the cascade-type system is obtained as:

$$
\begin{aligned}
& \dot{\rho}_{11}=\Gamma_{21} \rho_{22}-\frac{i}{2} \Omega_{p}\left(\rho_{12}-\rho_{21}\right), \\
& \dot{\rho}_{22}=-\Gamma_{21} \rho_{22}+\Gamma_{32} \rho_{33}-\frac{i}{2} \Omega_{p}\left(\rho_{21}-\rho_{12}\right)-\frac{i}{2} \Omega_{c}\left(\rho_{23}-\rho_{32}\right), \\
& \dot{\rho}_{33}=-\Gamma_{32} \rho_{33}-\frac{i}{2} \Omega_{c}\left(\rho_{32}-\rho_{23}\right),
\end{aligned}
$$




$$
\begin{aligned}
\dot{\rho}_{21} & =\bar{\gamma}_{21} \rho_{21}+\frac{i}{2} \Omega_{p}\left(\rho_{11}-\rho_{22}\right)+\frac{i}{2} \Omega_{c} \rho_{31}, \\
\dot{\rho}_{32} & =\bar{\gamma}_{32} \rho_{32}+\frac{i}{2} \Omega_{c}\left(\rho_{22}-\rho_{33}\right)-\frac{i}{2} \Omega_{p} \rho_{31}, \\
\dot{\rho}_{31} & =\bar{\gamma}_{31} \rho_{31}-\frac{i}{2} \Omega_{p} \rho_{32}+\frac{i}{2} \Omega_{c} \rho_{21},
\end{aligned}
$$

where $\bar{\gamma}_{21}=i \Delta_{p}-\gamma_{21}, \bar{\gamma}_{32}=i \Delta_{c}-\gamma_{32}$ and $\bar{\gamma}_{31}=i\left(\Delta_{p}-\Delta_{c}\right)-\gamma_{31} ; \Omega_{p}=d_{21} E_{p} / \hbar$ and $\Omega_{c}=d_{32} E_{c} / \hbar$ are the Rabi frequencies for the probe and coupling laser; $\Delta_{p}=\omega_{p}-\omega_{21}$ and $\Delta_{c}=\omega_{c}-\omega_{32}$ are the frequency detunings of the coupling and probe laser, respectively.

Using a similar approach to that shown in Sec. III.1, we found the solution of the matrix element $\rho_{21}$ for the probe field as follows:

$$
\begin{aligned}
& \rho_{21}= \frac{\left\{\begin{array}{l}
2 \bar{\gamma}_{12}\left(B_{123} B_{321}-B_{13} B_{31}\right) \\
\left.-\Omega_{c}\left(B B_{321}+B_{31} B-B_{23} B_{321}\right)\right] \\
\times i \Omega_{p}\left(B_{321} B_{123}-B_{31} B_{13}\right)
\end{array}\right]}{\Omega_{c}\left(B_{13} B_{23}-B_{13} B-B B_{123}\right)}-i \Omega_{p}\left(B_{123} B_{321}-B_{13} B_{31}\right) \\
& \Omega_{c}\left(B_{31} B_{32}-B_{31} B-B B_{321}\right)-\frac{\left[\begin{array}{l}
2 \bar{\gamma}_{12}\left(B_{123} B_{321}-B_{13} B_{31}\right) \\
\left.-\Omega_{c}\left(B B_{321}+B_{31} B-B_{23} B_{321}\right)\right] \\
\times\left[\bar{\gamma}_{21}\left(B_{321} B_{123}-B_{31} B_{13}\right)\right. \\
\left.-\Omega_{c}\left(B B_{123}+B_{13} B-B_{32} B_{123}\right)\right]
\end{array}\right\}}{\Omega_{c}\left(B_{13} B_{23}-B_{13} B-B B_{123}\right)}
\end{aligned}
$$

where $B_{321}=8 \Gamma_{32} \Gamma_{21} \bar{\gamma}_{32} \bar{\gamma}_{31}+2 \Gamma_{32} \Gamma_{21} \Omega_{p}^{2}-2 \Gamma_{21} \bar{\gamma}_{31} \Omega_{c}^{2}, \quad B=\Omega_{c}\left[\Gamma_{21} \Omega_{c}^{2}-\Gamma_{32} \Omega_{p}^{2}\right]$, $B_{32}=4 \Gamma_{32} \Gamma_{21} \bar{\gamma}_{32} \Omega_{c}, B_{13}=2 \Gamma_{21} \bar{\gamma}_{13} \Omega_{c}^{2}$, while $B_{k j i}$ and $\bar{\gamma}_{j i}$ are the complex conjugation of $B_{i j k}$ and $\bar{\gamma}_{i j}$, respectively.

We also apply to the case of ${ }^{85} \mathrm{Rb}$ atomic vapor with the states $|1\rangle,|2\rangle$ and $|3\rangle$ are [Authors: "are" should be deleted] chosen as $5 \mathrm{~S}_{1 / 2}(F=2), 5 \mathrm{P}_{3 / 2}(F=3 \prime)$ and $5 \mathrm{D}_{5 / 2}(F=4$ ?), respectively. In this case, the decay rates are $\Gamma_{21}=6 \gamma$ and $\Gamma_{32}=0.97 \gamma[21]$, the decoherence rate between states $|3\rangle$ and $|1\rangle$ is $\gamma_{31}=\left(\Gamma_{21}+\Gamma_{32}\right) / 2=3.5 \gamma$.

By choosing the parameters $\Omega_{c}=40 \gamma, \Delta_{c}=0$ and $C=1000 \gamma$, the surface plot of the behavior of output-input fields versus the frequency detuning of the probe laser is plotted in Fig. $7 \mathrm{a}$. Similarly, we set $\Delta_{p}=20 \gamma$, the surface plot of the behavior of output-input fields versus the intensity of the coupling laser is displayed in Fig. 7b. In comparison with the $\Lambda$-type system, we find that occurrence of OB with the values of Rabi frequency $\Omega_{c}$, frequency detuning $\Delta_{p}$ as well as cooperation parameter $C$ which are much larger. This means that the threshold intensity of OB curve in the cascade-type system is much stronger than that in the $\Lambda$-type system. Physical reason for this phenomenon is because of [due to the fact that] the decaying rate in three-level cascade system is larger than that in three-level $\Lambda$ system. However, we can reduce this decaying rate by choosing the uppermost excited states as the Rydberg states, which have life-time of few $\mu \mathrm{s}$ (life-time of the state $38 \mathrm{D}_{5 / 2}$ of Rb atom is $13 \mu \mathrm{s}$, see Ref. [22], for example). In this case, one may reduce the intensity thresholds of $\mathrm{OB}$ which can obtain is similar to three-level $\Lambda$ system. 

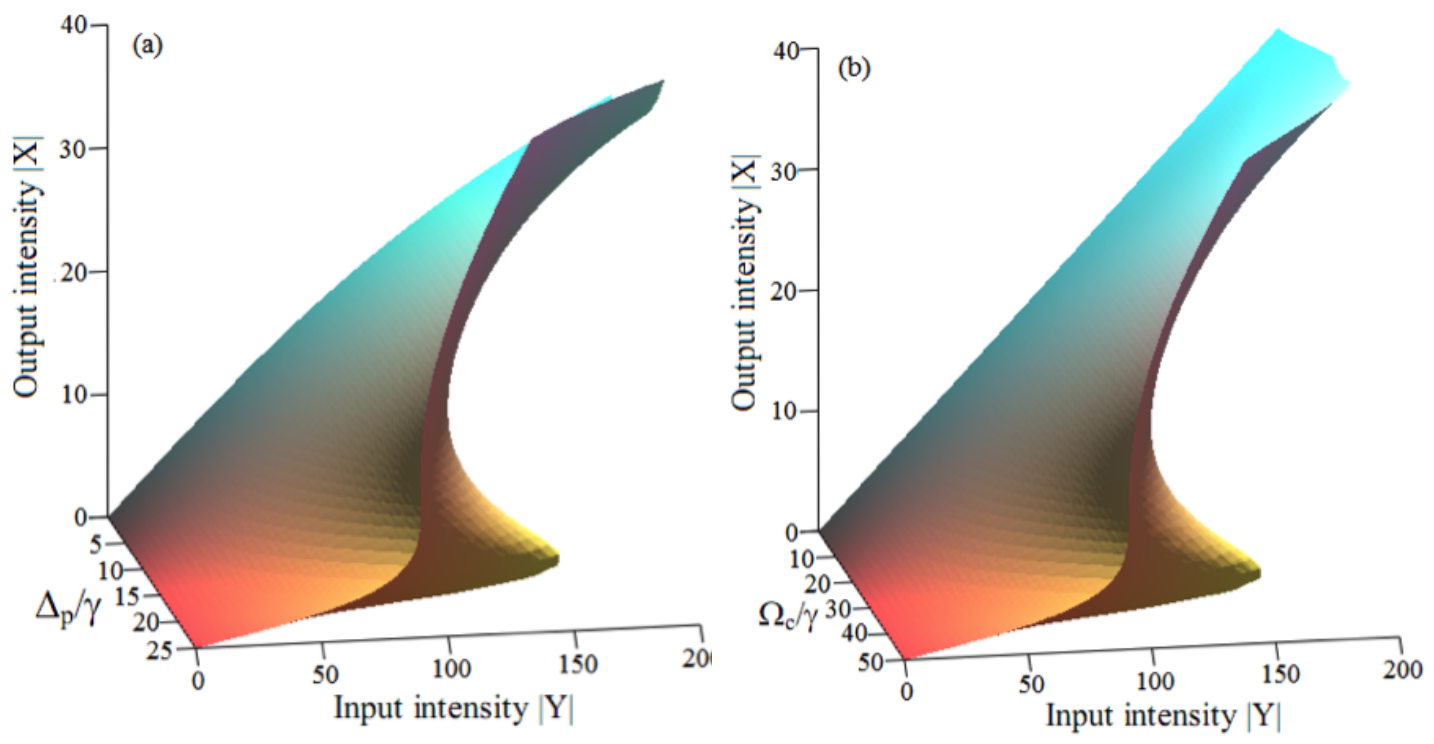

Fig. 7. (a) surface plots of input-output intensity relation versus frequency detuning $\Delta_{p}$ as $\Omega_{c}=40 \gamma$. (b) surface plots of input-output intensity relation versus Rabi frequency $\Omega_{c}$ as $\Delta_{p}=20 \gamma$. The other parameters are $\Delta_{c}=0$ and $C=1000 \gamma$.

\section{III.3. V-type system}

In this part, we will analyze the bistable behavior of output field intensity versus input field intensity in the V-type atomic system, as displayed in Fig. 2c. The density matrix equation for such atomic system can be derived as follows:

$$
\begin{aligned}
& \dot{\rho}_{11}=\Gamma_{31} \rho_{33}+\Gamma_{21} \rho_{22}+\frac{i}{2} \Omega_{p}\left(\rho_{21}-\rho_{12}\right)+\frac{i}{2} \Omega_{c}\left(\rho_{31}-\rho_{13}\right), \\
& \dot{\rho}_{22}=-\left(\Gamma_{21}+\Gamma_{23}\right) \rho_{22}+\frac{i}{2} \Omega_{p}\left(\rho_{12}-\rho_{21}\right), \\
& \dot{\rho}_{33}=-\Gamma_{31} \rho_{33}+\Gamma_{23} \rho_{22}+\frac{i}{2} \Omega_{c}\left(\rho_{13}^{-} \rho_{31}\right), \\
& \dot{\rho}_{21}=\bar{\gamma}_{21} \rho_{21}+\frac{i}{2} \Omega_{p}\left(\rho_{11}-\rho_{22}\right)-\frac{i}{2} \Omega_{c} \rho_{23}, \\
& \dot{\rho}_{31}=\bar{\gamma}_{31} \rho_{31}+\frac{i}{2} \Omega_{c}\left(\rho_{11}-\rho_{33}\right)-\frac{i}{2} \Omega_{p} \rho_{32}, \\
& \dot{\rho}_{23}=\bar{\gamma}_{23} \rho_{23}+\frac{i}{2} \Omega_{p} \rho_{13}-\frac{i}{2} \Omega_{c} \rho_{21},
\end{aligned}
$$

where $\bar{\gamma}_{21}=i \Delta_{p}-\gamma_{21}, \bar{\gamma}_{23}=i\left(\Delta_{p}-\Delta_{c}\right)-\gamma_{23}$ and $\bar{\gamma}_{31}=i \Delta_{c}-\gamma_{31} ; \Omega_{p}=d_{21} E_{p} / \hbar$ and $\Omega_{c}=d_{31} E_{c} / \hbar$ are the Rabi frequencies for the probe and coupling laser; $\Delta_{p}=\omega_{p}-\omega_{21}$ and $\Delta_{c}=\omega_{c}-\omega_{31}$ are the frequency detunings of the coupling and probe laser, respectively. 
Using a similar approach to that shown in Sec. III.1, the solution of the matrix element $\rho_{21}$ for the probe field can be derived as:

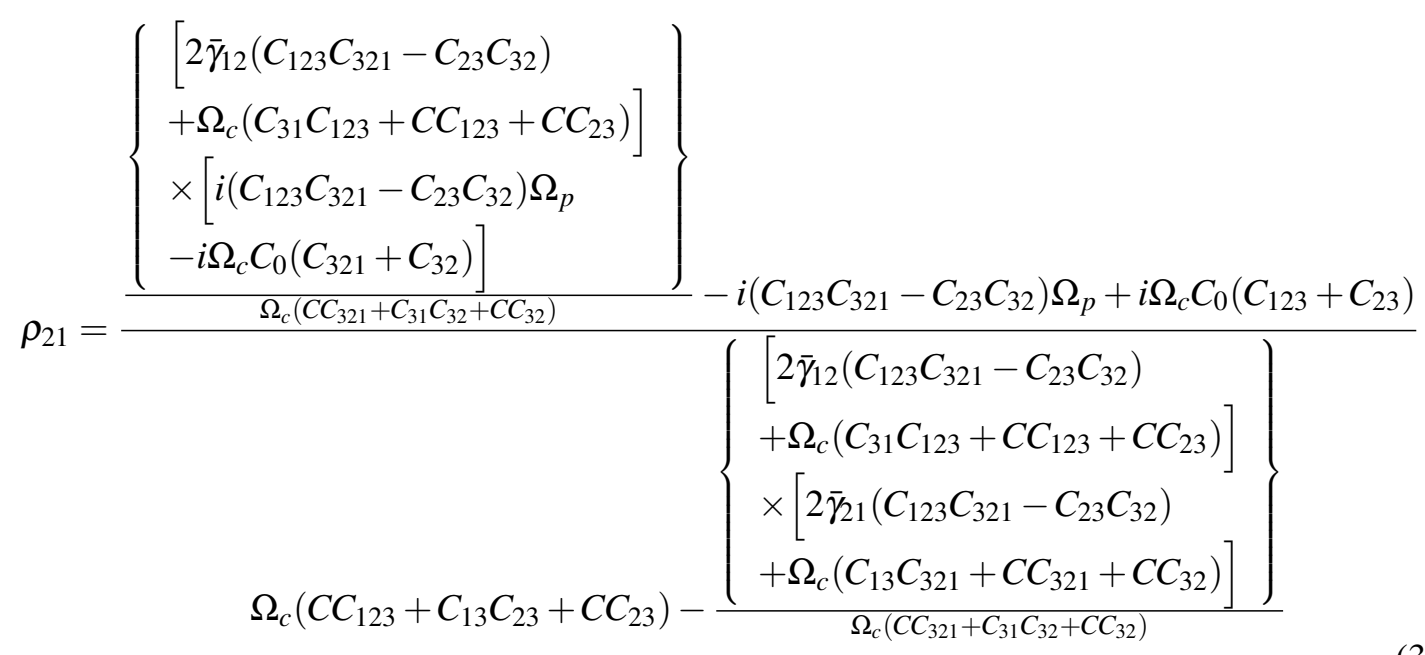

where: $C_{31}=4 \Gamma_{31}\left(\Gamma_{21}+\Gamma_{23}\right) \bar{\gamma}_{31} \Omega_{c}, C_{23}=4\left(\Gamma_{21}+\Gamma_{23}\right) \Omega_{c}^{2} \bar{\gamma}_{23}, C=\Omega_{c}\left[\left(\Gamma_{31}+2 \Gamma_{23}\right) \Omega_{p}^{2}-2\left(\Gamma_{21}+\right.\right.$ $\left.\left.\Gamma_{23}\right) \Omega_{c}^{2}\right] C_{321}=8 \Gamma_{31}\left(\Gamma_{21}+\Gamma_{23}\right) \bar{\gamma}_{32} \bar{\gamma}_{31}+2 \Gamma_{31}\left(\Gamma_{21}+\Gamma_{23}\right) \Omega_{p}^{2}-4\left(\Gamma_{21}+\Gamma_{23}\right) \bar{\gamma}_{32} \Omega_{c}^{2}, C_{0}=2 \Gamma_{31}\left(\Gamma_{21}+\right.$ $\left.\Gamma_{23}\right) \Omega_{p} \Omega_{c}$, and $C_{k j i}$ and $\bar{\gamma}_{j i}$ are the complex conjugation of $C_{i j k}$ and $\bar{\gamma}_{i j}$, respectively.
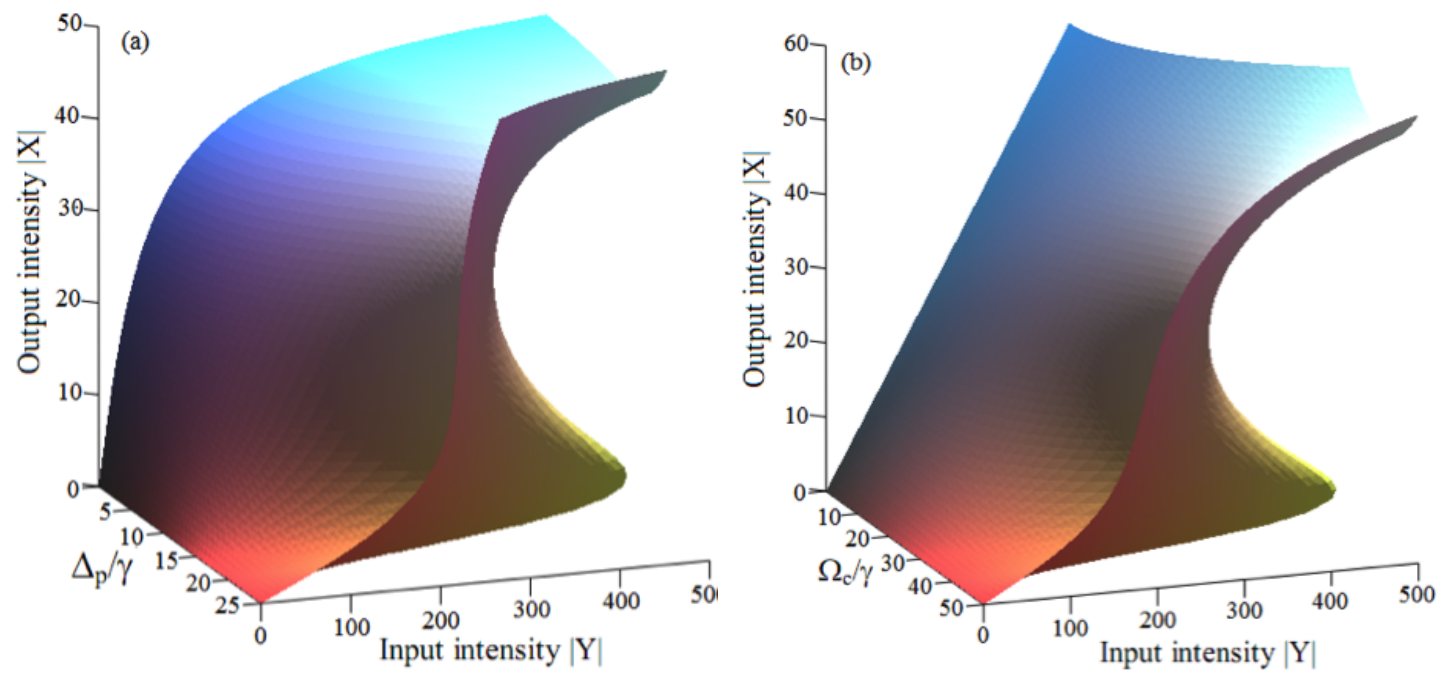

Fig. 8. (a) surface plots of input-output intensity relation versus frequency detuning $\Delta_{p}$ as $\Omega_{c}=40 \gamma$. (b) surface plots of input-output intensity relation versus Rabi frequency $\Omega_{c}$ as $\Delta_{p}=20 \gamma$. The other parameters are $\Delta_{c}=0$ and $C=1000 \gamma$.

In this scheme, the states $|1\rangle,|2\rangle$ and $|3\rangle$ are chosen as $5 S_{1 / 2}(F=2), 5 P_{3 / 2}\left(F^{\prime}=3\right)$ and $5 P_{3 / 2}\left(F=2^{\prime \prime}\right)$ of ${ }^{85} \mathrm{Rb}$ atom, respectively. The decay rates are $\Gamma_{21}=6 \gamma$ and $\Gamma_{31}=6 \gamma$ [21], the decaying rate between states $|2\rangle$ and $|3\rangle$ as $\gamma_{23}=\left(\Gamma_{21}+\Gamma_{31}\right) / 2=6 \gamma$. Fig. 8a shows the surface 
plot of the behavior of output-input fields versus the frequency detuning of the probe laser when $\Omega_{c}=40 \gamma, \Delta_{c}=0$ and $C=1000 \gamma$. Fig. 8b shows the surface plot of the behavior of outputinput fields versus the intensity of the coupling laser when $\Delta_{p}=20 \gamma, \Delta_{c}=0$ and $C=1000 \gamma$. In comparison with the cascade-type system, we find that under the same parameters the switching thresholds here are much larger.

\section{CONCLUSIONS}

We have made a comparative study of OB in the three-level configurations under EIT condition. Due to zero absorption and giant Kerr nonlinearity of the EIT medium, the threshold intensity and width of the OB is much less than those of the traditional ones. In addition, we found that threshold intensity of OB in $\Lambda$-type system is much less than that in the other ones, whereas that in $\mathrm{V}$-type system is the strongest. A key reason of low threshold intensity in the three-level $\Lambda$ type system is due to the smallest decaying rate. The analytical results are convenient to study applications in photonic devices working at low-light intensity.

\section{ACKNOWLEDGMENT}

The financial support from Ministry of Science and Technology through the grant code ĐTĐLCN.17/17 is acknowledged.

\section{REFERENCES}

[1] E. Abraham and S. D. Smith, Rep. Prog. Phys. 45 (1982) 815-885.

[2] L.A. Lugiato, "Theory of Optical Bistability", in E. Wolf (ed.), Progress in Optics, Vol. 21, North Holland, Amsterdam 1984, pp. 71-216.

[3] H.M. Gibbs, Optical Bistability: Controlling Light with Light, Academic Press, New York, 1985.

[4] K.J. Boller, A. Imamoglu, S.E. Harris, Phys. Rev. Lett. 66 (1991) 2593.

[5] L. V. Doai, P. V. Trong, D. X. Khoa, and N. H. Bang, Optik 125 (2014) 3666.

[6] D. X. Khoa, P. V. Trong, L. V. Doai and N. H. Bang, Phys. Script. 91 (2016) 035401.

[7] D. X. Khoa, L. C. Trung, P. V. Thuan, L. V. Doai and N. H. Bang, J. Opt. Soc. Am. B 34 (2017) 1255.

[8] H. Wang, D. Goorskey, and M. Xiao, Phys. Rev. Lett. 87 (2001) 073601.

[9] D. X. Khoa, L. V. Doai, D. H. Son, and N. H. Bang, J. Opt. Soc. Am. B 31 (2014) 1330.

[10] L. V. Doai, D. X. Khoa and N. H. Bang, Phys. Scr. 90 (2015) 045502.

[11] S.Q. Gong, S.D. Du, Z.Z. Xu, and S.H. Pan, Phys. Lett. A 222 (1996) 237.

[12] Hai Wang, D. J. Goorskey, and Min Xiao, Phys. Rev A 65 (2001) 011801R.

[13] A. Joshi, W. Yang and M. Xiao, Phys. Lett. A 315 (2003) 203.

[14] A. Joshi, W. Yang and M. Xiao, , Phys. Rev. A. 68 (2003) 015806.

[15] D. Cheng, C. Liu and S. Gong, Phys. Lett. A 332 (2004) 244.

[16] A. Joshi and M. Xiao, Phys. Rev. Lett. 91 (2003) 143904.

[17] A. Joshi, A. Brown, H. Wang, and M. Xiao, Phys. Rev. A 67 (2003) 041801(R).

[18] J. Li, Physica D 228 (2007) 148.

[19] Z. Wang, A-X. Chen, Y. Bai, W.-X. Yang and R.-K. Lee, J. Opt. Soc. Am. B 29 (2012) 2891.

[20] A. Joshi, M. Xiao, Controlling steady-state and dynamical properties of atomic optical bistability, World Scientific Publishing, 2012.

[21] Daniel Adam Steck, $\mathrm{Rb}^{85}$ D Line Data: http://steck.us/alkalidata

[22] M. Mack, J. Grimmel, F. Karlewski, L. Sárkány, H. Hattermann and J. Fortágh, Phys. Rev. A 92 (2015) 012517. 